\title{
Prescribing inner parts of derivatives of inner functions
}

\author{
Oleg Ivrii
}

August 8, 2017

\begin{abstract}
Let $\mathscr{J}$ be the set of inner functions whose derivative lies in Nevanlinna class. In this note, we show that the natural map

$$
F \rightarrow \operatorname{Inn}\left(F^{\prime}\right) \quad: \quad \mathscr{J} / \operatorname{Aut}(\mathbb{D}) \rightarrow \operatorname{Inn} / S^{1}
$$

is injective but not surjective. More precisely, we show that that the image consists of all inner functions of the form $B S_{\mu}$ where $B$ is a Blaschke product and $S_{\mu}$ is the singular factor associated to a measure $\mu$ whose support is contained in a countable union of Beurling-Carleson sets. Our proof is based on extending the work of D. Kraus and O. Roth on maximal Blaschke products to allow for singular factors. This answers a question raised by K. Dyakonov.
\end{abstract}

\section{Introduction}

Consider the following curious differentiation procedure: to a Blaschke product

$$
F(z)=\prod_{i=1}^{d} \frac{z-a_{i}}{1-\overline{a_{i}} z}
$$

of degree $d \geq 1$, one can associate a Blaschke product $B$ of degree $d-1$ whose zeros are located at the critical points of $F$ (that is, at the zeros of $F^{\prime}$ ). It is a classical result of M. Heins [10, Section 29] that this correspondence is a bijection, provided 
one considers $F$ modulo post-composition with Möbius transformations (as not to change its critical set) and $B$ up to rotations (which preserve the zero set).

In this paper, we discuss an infinite degree analogue of this problem posed by K. Dyakonov in [8, 9]. We need some definitions. An inner function is a holomorphic self-map of the unit disk such that for almost every $\theta \in[0,2 \pi)$, the radial limit $\lim _{r \rightarrow 1} F\left(r e^{i \theta}\right)$ exists and has absolute value 1 . Let Inn denote the space of all inner functions. We will also be concerned with the subclass $\mathscr{J}$ of inner functions whose derivative lies in Nevanlinna class, i.e. which satisfy

$$
\lim _{r \rightarrow 1} \frac{1}{2 \pi} \int_{0}^{2 \pi} \log ^{+}\left|F^{\prime}\left(r e^{i \theta}\right)\right| d \theta<\infty .
$$

Together with Jensen's formula, (1.1) implies that the set of critical points $\left\{c_{i}\right\}$ of $F$ satisfies the Blaschke condition $\sum\left(1-\left|c_{i}\right|\right)<\infty$, and is therefore the zero set of some Blaschke product, which could be either finite or infinite.

According to the work of Ahern and Clark, if $F^{\prime}$ is a Nevanlinna class function, then it admits an "inner-outer" decomposition $F^{\prime}=\operatorname{Inn} F^{\prime}$. Out $F^{\prime}$, see Lemma 3.2 below. The mapping $F \rightarrow \operatorname{Inn} F^{\prime}$ from $\mathscr{J}$ to Inn generalizes the construction outlined for finite Blaschke products above, however, in addition to recording the critical set of $F$, Inn $F^{\prime}$ may also contain a non-trivial singular factor. This feature allows us to distinguish different Blaschke products with the same critical set. In this paper, we prove the following theorem:

Theorem 1.1. Let $\mathscr{J}$ be the set of inner functions whose derivative lies in Nevanlinna class. The natural map

$$
F \rightarrow \operatorname{Inn}\left(F^{\prime}\right) \quad: \quad \mathscr{J} / \operatorname{Aut}(\mathbb{D}) \rightarrow \operatorname{Inn} / S^{1}
$$

is injective. The image consists of all inner functions of the form $B S_{\mu}$ where $B$ is a Blaschke product and $S_{\mu}$ is the singular factor associated to a measure $\mu$ whose support is contained in a countable union of Beurling-Carleson sets.

In [8], K. Dyakonov showed that $\operatorname{Inn} F^{\prime}$ is trivial if and only if $F$ is a Möbius transformation. After reading Dyakonov's work, the author realized that a theorem of D. Kraus can be reformulated as " $F \rightarrow \operatorname{Inn} F$ ' is a bijection from Maximal Blaschke Products in $\mathscr{J}$ to the space of all Blaschke Products." The main focus of this paper will be to understand the role of singular factors. 


\section{$1.1 \quad$ Strategy}

We now state several propositions which will be used to show Theorem 1.1. These will be proved in Sections 5 and 6 after we develop the necessary tools.

Lemma 1.2 (Decomposition rule). An inner function $B_{C} S_{\mu}$ lies in the image of $F \rightarrow \operatorname{Inn} F^{\prime}$ if and only if its singular part $S_{\mu}$ does.

Therefore, to describe the image of our mapping, it suffices to determine which singular inner functions $S_{\mu}$ can be represented as $S_{\mu}=\operatorname{Inn} F_{\mu}^{\prime}$ with $F_{\mu} \in \mathscr{J}$. If such an $F_{\mu}$ can be found (which is necessarily unique), we say that the measure $\mu$ is constructible.

Lemma 1.3 (Product rule). Suppose measures $\mu_{j}, j=1,2, \ldots$ are constructible. If their sum $\mu=\sum_{j=1}^{\infty} \mu_{j}$ is finite, then $\mu$ is also constructible.

Lemma 1.4 (Division rule). If a measure $\mu$ is constructible, then any $\nu \leq \mu$ is also constructible.

Recall that a Beurling-Carleson set is a closed subset of the unit circle of zero Lebesgue measure whose complement is a union of arcs $\bigcup_{k} I_{k}$ with $\sum\left|I_{k}\right| \log \frac{1}{\left|I_{k}\right|}<\infty$. To obtain a large supply of constructible measures, we use the following result of Cullen [3]:

Lemma 1.5. Suppose the support of $\mu$ is contained in a Beurling-Carleson set. Then $S_{\mu}^{\prime} \in \mathcal{N}$

Since $S_{\mu}$ divides $S_{\mu}^{\prime}$, the division rule implies that any measure $\mu$ supported on a Beurling-Carleson set is constructible. By the product rule, any measure supported on a countable union of Beurling-Carleson sets is also constructible. Theorem 1.1 states that any constructible measure is of this form. Moreover, Theorem 1.1 implies that Cullen's theorem is essentially sharp:

Corollary 1.6. Suppose $\mu$ is a measure on the unit circle with $S_{\mu}^{\prime} \in \mathcal{N}$. Then, the support of $\mu$ is contained in a countable union of Beurling-Carleson sets. 
On the other side of the spectrum, we have invisible measures. We say that a finite positive singular measure $\mu$ is invisible if for any measure $0<\nu \leq \mu$, there does not exist a function $F_{\nu} \in \mathscr{J}$ with $\operatorname{Inn} F_{\nu}^{\prime}=S_{\nu}$. In Section 5, we will show that any singular measure on the unit circle $\mu$ can be uniquely decomposed into a constructible part and an invisible part: $\mu=\mu_{\mathrm{con}}+\mu_{\text {inv }}$. To complete the proof of Theorem 1.1, we give a criterion for a measure to be invisible:

Theorem 1.7. Suppose $\mu$ is a measure on the unit circle which does not charge Beurling-Carleson sets. Then, it is invisible.

The reader may notice that the notion of an invisible measure coincides with the description of cyclic inner functions in Bergman spaces given independently by Korenblum [11] and Roberts [19]. To prove Theorem 1.7, we will first show that any measure $\mu$ with modulus of continuity $\omega(t) \leq C t \log (1 / t)$ is invisible. To obtain the full result, we use an iterative scheme based on a clever decomposition of a measure that does not charge Beurling-Carleson sets into " $t \log 1 / t$ "-pieces from [19].

We conclude the introduction by spending a moment to check that the map in Theorem 1.1 is well-defined:

Lemma 1.8. If $F \in \mathscr{J}$ is an inner function, then for any Möbius transformation $T \in$ Aut $\mathbb{D}$, the Frostman shift $T \circ F \in \mathscr{J}$ and $\operatorname{Inn}(T \circ F)^{\prime}=\operatorname{Inn} F^{\prime}$.

Proof. From the chain rule, we have $(T \circ F)^{\prime}(z)=T^{\prime}(F(z)) \cdot F^{\prime}(z)$. Since $\log \left|T^{\prime}\right|$ is bounded, $T \circ F \in \mathscr{J}$. The equality also tells us that the inner part $\operatorname{Inn}(T \circ F)^{\prime}$ is divisible by $\operatorname{Inn} F^{\prime}$. Using $T^{-1}$ in place of $T$, we see that $\operatorname{Inn} F^{\prime}$ is divisible by $\operatorname{Inn}(T \circ F)^{\prime}$. Hence, $\operatorname{Inn}(T \circ F)^{\prime}=\operatorname{Inn} F^{\prime}$ agree (up to a unimodular constant).

\subsection{Notation}

Let $m$ denote the Lebesgue measure on $\mathbb{S}^{1}$, normalized to have unit mass and $\lambda_{\mathbb{D}}=$ $\frac{|d z|}{1-|z|^{2}}$ be the Poincaré metric on the unit disk. For a holomorphic mapping $F: \mathbb{D} \rightarrow$ $\mathbb{D}$, we denote the associated conformal metric of constant curvature -4 by

$$
\lambda_{F}:=\frac{\left|F^{\prime}\right|}{1-|F|^{2}} .
$$


Given a Blaschke sequence $C$ in the unit disk, let $B_{C}$ be the Blaschke product with zero set $C$ and $F_{C}$ denote the maximal Blaschke product with critical set $C$. In order for $B_{C}$ and $F_{C}$ to be uniquely defined, we use the normalizations $B_{C}(1)=1$ and $F_{C}(0)=0, F_{C}^{\prime}(0)>0$ (or $F_{C}^{(n+1)}(0)>0$ if $C$ contains 0 with multiplicity $n$ ). For a singular measure $\mu$ on the unit circle, we let $S_{\mu}$ be the associated singular inner function.

\section{Background on conformal metrics}

Given an at most countable set $C$ in the unit disk (counted with multiplicity), the machinery of Kraus and Roth [12]-[17] seeks to construct a Blaschke product with critical set $C$. If such a Blaschke product does not exist, then the machinery does not produce anything. If there are Blaschke products with critical set $C$, the machinery produces the optimal or maximal Blaschke product $F_{C}$.

Instead of constructing $F_{C}$ directly, Kraus and Roth construct the conformal metric $F_{C}^{*} \lambda_{\mathbb{D}}$ - the pullback of the Poincaré metric on the disk. We give a brief overview of their construction. Following Heins, an SK-metric $\lambda(z)|d z|$ is a conformal pseudometric on a domain $U$ whose density $\lambda: U \rightarrow[0, \infty)$ is a continuous function with curvature

$$
k_{\lambda}=-\frac{\Delta \log \lambda}{\lambda^{2}} \leq-4
$$

in the sense of distributions. According to [10, Section 13] or [15, Definition 4.11], a collection $\Phi$ of SK-metrics is a Perron family if it is closed under modifications and taking maxima. The first condition means that given a round disk $D \subset U$ and a metric $\lambda \in \Phi$, the (unique) SK-metric $M_{D} \lambda$ which agrees with $\lambda$ on $U \backslash D$ and has curvature -4 in $D$ lies in $\Phi$; while the second condition says that for any $\lambda_{1}, \lambda_{2} \in \Phi$, their pointwise maximum $\max \left(\lambda_{1}, \lambda_{2}\right)$ is also in $\Phi$. Heins proved that if a Perron family is non-empty, then the supremum of all metrics in $\Phi$ is a regular conformal metric of curvature -4 , where regular means " $C^{2}$ on the complement of $\{z \in U: \lambda(z)=0\}$."

We also recall a complementary theorem due to Liouville [14, Theorem C] which says that if a conformal metric $\lambda(z)|d z|$ has constant curvature -4 and all its zeros 
$c_{i}$ have integral multiplicities, that is if

$$
\lim _{z \rightarrow c_{i}} \frac{\lambda(z)}{\left|z-c_{i}\right|^{m_{i}}}=L_{i}, \quad \text { for some } \quad 1 \leq m_{i} \in \mathbb{Z}, \quad 0<L_{i}<\infty,
$$

then $\lambda(z)|d z|$ is necessarily of the form $\lambda_{F}=F^{*} \lambda_{\mathbb{D}}$ for some holomorphic function $F: U \rightarrow \mathbb{D}$. Furthermore, the function $F$ is unique up to post-composition with a Möbius transformation.

For a set $C$ in the unit disk, let $\Phi_{C}$ be the collection of all conformal metrics vanishing on $C$. It clearly verifies the two axioms of being a Perron family on the domain $\mathbb{D} \backslash C$. Provided $\Phi_{C}$ is non-empty, one obtains a metric of constant curvature -4 and a holomorphic function $F_{C}$ which vanishes on $C$ to the correct order. Leveraging the maximality of the metric $\lambda_{F_{C}}$, Kraus [12] proved that the outer and singular inner factors of $F_{C}$ are trivial. In other words, $F_{C}$ is a Blaschke product.

In the case when the critical set $C$ is a Blaschke sequence, Kraus made the fundamental observation that $\left|B_{C}\right| \lambda_{\mathbb{D}}$ is an SK-metric which guarantees that the Perron family $\Phi_{C}$ is non-empty. (More generally, given a holomorphic function $H$ with $\|H\|_{\infty} \leq 1$ and a metric $\lambda$ of curvature $-4,|H| \cdot \lambda$ is an SK-metric.)

Further exploiting the lower bound $\lambda_{F_{C}} \geq\left|B_{C}\right| \lambda_{\mathbb{D}}$, Kraus obtained the following remarkable result [12, Theorem 4.4]:

Theorem 2.1 (Kraus). Suppose $C$ is a Blaschke sequence in the disk and $\lambda$ is a metric of constant curvature -4 which vanishes precisely at $C$ with the correct multiplicity. Then $\lambda=\lambda_{F_{C}}$ if and only if

$$
\lim _{r \rightarrow 1} \int_{|z|=r} \log \frac{\lambda}{\lambda_{\mathbb{D}}} d \theta=0 .
$$

In Section 3, we will use ideas of Ahern and Clark to show that the above theorem can be alternatively formulated as:

Corollary 2.2. Suppose $C$ is a Blaschke sequence in the disk. An infinite Blaschke product $F \in \mathscr{J}$ is the maximal Blaschke product associated to $C$ if and only if the singular factor of $\operatorname{Inn} F^{\prime}$ is trivial, i.e. if $\operatorname{Inn} F^{\prime}=B_{C}$. 
In order to generalize the arguments of Kraus and Roth to allow for singular factors, we will need:

Lemma 2.3 (Fundamental Lemma). For any inner function $F \in \mathscr{J}$,

$$
\lambda_{F} \geq\left|\operatorname{Inn} F^{\prime}\right| \lambda_{\mathbb{D}} .
$$

In fact, $\lambda_{F}$ is the smallest metric of constant curvature -4 with this property.

Note that the minimality of the metric $\lambda_{F}$ implies that the map $F \rightarrow \operatorname{Inn} F^{\prime}$ from Theorem 1.1 is injective. As explained above, the inequality (2.2) holds for maximal Blaschke products. In Section 4 , we will deduce the general case by considering finite approximations.

Using the factorization $F^{\prime}=\operatorname{Inn} F^{\prime}$. Out $F^{\prime}$, one can rewrite (2.2) as

$$
\frac{1-|F(z)|}{1-|z|} \leq \mid \text { Out } F^{\prime} \mid,
$$

which was first proved by Dyakonov in [6, Theorem 2.1] using Julia's lemma. The reader may also consult [7, Corollary 2.1] for additional remarks. In view of the above discussion, the fundamental lemma may be viewed as a refinement of Dyakonov's theorem.

\subsection{Wedge of two metrics}

Given two inner functions $F, G \in \mathscr{J}$, consider the family $\Phi_{F, G}$ of SK-metrics that are pointwise less than $\min \left(\lambda_{F}, \lambda_{G}\right)$. This family is not empty: the metric $\left|\operatorname{Inn} F^{\prime}\right|$. $\left|\operatorname{Inn} G^{\prime}\right| \cdot \lambda_{\mathbb{D}}$ is in it, as Lemma 2.3 shows. Taking the supremum of conformal metrics in $\Phi_{F, G}$, we get a regular conformal metric $\lambda_{F \wedge G}$ of constant curvature -4 . Therefore, it is the pullback of $\lambda_{\mathbb{D}}$ by a holomorphic function which we denote $H=F \wedge G$.

To see that the outer part of $H$ is trivial, i.e. that $H$ is inner, we can use the clever argument of Kraus [12, Proof of Theorem 1.2]. The relevant equation here is:

$$
\left[\frac{\left|H^{\prime}\right|}{|H| \log \frac{1}{|H|}} \cdot|\operatorname{Inn} H|\right] \cdot\left|\operatorname{Inn} F^{\prime}\right| \cdot\left|\operatorname{Inn} G^{\prime}\right| \leq \frac{\left|H^{\prime}\right|}{1-|H|^{2}},
$$


where the expression in the square brackets is bounded above by $\lambda_{\mathbb{D}}$ since it is an SK-metric (see [12, Lemma 2.17]). One finds a contradiction by examining the behaviour of both sides as $z \rightarrow e^{i \theta}$ radially to a point on the unit circle at which $\mid$ Out $H\left(e^{i \theta}\right) \mid<1$ and $\left|\operatorname{Inn} H\left(e^{i \theta}\right)\right|=\left|\operatorname{Inn} F^{\prime}\left(e^{i \theta}\right)\right|=\left|\operatorname{Inn} G^{\prime}\left(e^{i \theta}\right)\right|=1$.

\subsection{Hull of a conformal metric}

For an SK-metric $\kappa$, let $\Psi_{\kappa}$ be the collection of all metrics of constant curvature -4 which are greater than $\kappa$ and $\Phi_{\kappa}$ be the collection of all SK-metrics that are less than all metrics in $\Psi_{\kappa}$. Since $\Phi_{\kappa}$ is a Perron family, its supremum is a metric $\hat{\kappa}$ of curvature -4 . We call $\hat{\kappa}$ the hull of $\kappa$. From the definition, it is clear that $\hat{\kappa}$ is the smallest metric of curvature -4 which exceeds $\kappa$. In this terminology, Lemma 2.3 says that $\lambda_{F}$ is the hull of $\left|\operatorname{Inn} F^{\prime}\right| \lambda_{\mathbb{D}}$.

\section{Gap of a Nevanlinna function}

By definition, the Nevanlinna class $\mathcal{N}$ consists of holomorphic functions on the unit disk for which

$$
\sup _{0<r<1} \frac{1}{2 \pi} \int_{|z|=r} \log ^{+}|f(z)| d \theta<\infty,
$$

see for instance [5]. It is well known that (unless $f$ is identically zero) this condition is equivalent to the boundedness of

$$
\sup _{0<r<1} \frac{1}{2 \pi} \int_{|z|=r}|\log | f(z)|| d \theta
$$

Since $\log |f(z)|$ is a subharmonic function, $\lim _{r \rightarrow 1} \frac{1}{2 \pi} \int_{|z|=r} \log |f(z)| d \theta$ exists and is finite. However, unlike the Hardy norms, it need not be the case that

$$
\lim _{r \rightarrow 1} \frac{1}{2 \pi} \int_{|z|=r} \log |f(z)| d \theta=\frac{1}{2 \pi} \int_{|z|=1} \log |f(z)| d \theta
$$

where in the integral in the right hand side, we consider the radial boundary values of $f$ which are known to exist a.e. To understand the cause of the discrepancy, we 
consider the canonical decomposition of $f=B\left(S / S_{1}\right) O$ into a Blaschke product, a quotient of singular inner functions and an outer function:

$$
\begin{aligned}
B & =\prod_{i}-\frac{\overline{a_{i}}}{\left|a_{i}\right|} \cdot \frac{z-a_{i}}{1-\overline{a_{i}} z} \\
S / S_{1} & =\exp \left(-\int_{\mathbb{S}^{1}} \frac{\zeta+z}{\zeta-z} d \sigma_{\zeta}\right), \quad \sigma \perp m, \\
O & =\exp \left(\int_{\mathbb{S}^{1}} \frac{\zeta+z}{\zeta-z} \log |f(\zeta)| d m_{\zeta}\right) .
\end{aligned}
$$

Given an interval $I$ on the unit circle, let $r I$ denote its radial projection onto the circle $S_{r}=\{z:|z|=r\}$. Fubini's theorem and the dominated converge theorem show:

\section{Lemma 3.1.}

$$
\operatorname{gap}(f):=\frac{1}{2 \pi} \int_{|z|=1} \log |f(z)| d \theta-\lim _{r \rightarrow 1}\left\{\frac{1}{2 \pi} \int_{|z|=r} \log |f(z)| d \theta\right\}=\sigma\left(\mathbb{S}^{1}\right) .
$$

More generally, if I is an interval on the unit circle,

$$
\operatorname{gap}_{I}(f):=\frac{1}{2 \pi} \int_{I} \log |f(z)| d \theta-\lim _{r \rightarrow 1}\left\{\frac{1}{2 \pi} \int_{r I} \log |f(z)| d \theta\right\}=\sigma(I),
$$

provided the endpoints of I do not charge $\sigma$.

\subsection{Applications to inner functions}

We now apply Lemma 3.1 to study inner functions with derivative in Nevanlinna class. We first give a slightly different perspective on a classical theorem due to Ahern and Clark:

Lemma 3.2 (Ahern-Clark). For an inner function $F \in \mathscr{J}$, its derivative admits a $B S O$ decomposition. In other words, the singular measure $\sigma\left(F^{\prime}\right) \geq 0$.

Proof. By Lemma 1.8, it suffices to consider the case when $F(0)=0$. Then $\left|F^{\prime}(x)\right| \geq$ 1 on the unit circle, e.g. see [18, Theorem 4.15]. In view of the fundamental inequality

$$
\left|F^{\prime}(r x)\right| \leq 4\left|F^{\prime}(x)\right|, \quad x \in \mathbb{S}^{1}, \quad 0<r<1,
$$


of Ahern and Clark [1], the dominated convergence theorem shows

$$
\int_{I} \log ^{+}\left|F^{\prime}(z)\right| d m-\lim _{r \rightarrow 1} \int_{r I} \log ^{+}\left|F^{\prime}(z)\right| d m=0,
$$

for any interval $I \subset \mathbb{S}^{1}$. However, by Fatou's lemma, the negative part of the logarithm can only dissipate and therefore

$$
\operatorname{gap}_{I}\left(F^{\prime}\right)=\int_{I} \log \left|F^{\prime}(z)\right| d m-\lim _{r \rightarrow 1} \int_{r I} \log \left|F^{\prime}(z)\right| d m \geq 0 .
$$

This completes the proof.

The following lemma relates the notions $\operatorname{gap}\left(F^{\prime}\right)$ and $\lambda_{F}$ :

Lemma 3.3. Let $I \subset \mathbb{S}^{1}$ be an interval. If $F \in \mathscr{J}$ then

$$
\frac{1}{2 \pi} \int_{I} \log \left|F^{\prime}(z)\right| d \theta=\lim _{r \rightarrow 1} \frac{1}{2 \pi} \int_{r I} \log \frac{1-|F(z)|^{2}}{1-|z|^{2}} d \theta .
$$

Proof. From the contraction of the hyperbolic distance $d_{\mathbb{D}}(F(0), F(z)) \leq d_{\mathbb{D}}(0, z)$, it follows that the quotient $\frac{1-|F(z)|^{2}}{1-|z|^{2}} \geq c_{F(0)}$ is bounded below by a positive constant. By the Schwarz lemma,

$$
\frac{1}{2 \pi} \int_{r I} \max \left(\log \left|F^{\prime}(z)\right|, \log c_{F(0)}\right) d \theta \leq \frac{1}{2 \pi} \int_{r I} \log \frac{1-|F(z)|^{2}}{1-|z|^{2}} d \theta .
$$

Applying the dominated convergence theorem like in the proof of Lemma 3.2 gives the $\leq$ inequality in $(3.2)$. For the $\geq$ direction, we average Dyakonov's inequality (2.3) over $z \in r I$ :

$$
\frac{1}{2 \pi} \int_{r I} \log \left|\operatorname{Out} F^{\prime}(z)\right| d \theta \geq \frac{1}{2 \pi} \int_{r I} \log \frac{1-|F(z)|^{2}}{1-|z|^{2}} d \theta .
$$

The lemma follows after taking $r \rightarrow 1$ since $\log \mid$ Out $F^{\prime}(z) \mid$ is the harmonic extension of $\log \left|F^{\prime}(z)\right|$ considered as a function on the unit circle.

The reader may compare the above lemma with [2, Theorem 3]. 


\subsection{Applications to conformal metrics}

Lemma 3.4. Suppose $F \in \mathscr{J}$ is an inner function for which

$$
\lambda_{F} \geq\left|B_{C} S_{\mu}\right| \cdot \lambda_{\mathbb{D}}
$$

Then, the singular measure $\sigma\left(F^{\prime}\right) \leq \mu$.

Proof. Let $I \subset \mathbb{S}^{1}$ be an interval. From the definition of $\lambda_{F}$,

$$
\int_{r I} \log \frac{\lambda_{F}}{\left|B_{C} S_{\mu}\right| \lambda_{\mathbb{D}}} d m=\int_{r I} \log \left(\frac{\left|F^{\prime}\right|\left(1-|z|^{2}\right)}{1-|F|^{2}}\right) d m-\int_{r I} \log \left|B_{C} S_{\mu}\right| d m .
$$

By Lemmas 3.1 and 3.3 , as $r \rightarrow 1$, this tends to

$$
0 \leq-\sigma\left(F^{\prime}\right)(I)+\sigma\left(S_{\mu}\right)(I),
$$

at least if $I$ is generic (there are extra terms if the endpoints of $I$ charge any of these singular measures).

Remark. The same conclusion holds under the seemingly weaker assumption $\lambda_{F} \geq$ $\left|B_{C} S_{\mu} O_{h}\right|$ where

$$
O_{h}=\exp \left(\int_{\mathbb{S}^{1}} \frac{\zeta+z}{\zeta-z} h(\zeta) d m_{\zeta}\right), \quad h: \mathbb{S}^{1} \rightarrow \mathbb{R},
$$

is an arbitrary outer function: the above computation results in $\sigma\left(F^{\prime}\right) \leq \mu-h d m$. Since $\sigma\left(F^{\prime}\right) \perp h d m$ are mutually singular, we have $\sigma\left(F^{\prime}\right) \leq \mu$ and $h \leq 0$.

Similar considerations show:

Lemma 3.5. If $F, G \in \mathscr{J}$ and the interval $I \subset \mathbb{S}^{1}$ is generic for both $\sigma\left(F^{\prime}\right)$ and $\sigma\left(G^{\prime}\right)$, then

$$
\lim _{r \rightarrow 1} \int_{|z|=r} \log \left(\lambda_{F} / \lambda_{G}\right) d m=-\sigma\left(F^{\prime}\right)(I)+\sigma\left(G^{\prime}\right)(I) .
$$

In particular, if $\lambda_{F} \geq \lambda_{G}$ then $\sigma\left(F^{\prime}\right) \leq \sigma\left(G^{\prime}\right)$.

Combining the above lemma with Theorem 2.1 gives Corollary 2.2 .

Lemma 3.6. If $\lambda_{G}$ is a metric of curvature -4 such that $\lambda_{G} \geq|H| \lambda_{\mathbb{D}}$ for some bounded holomorphic function $H \not \equiv 0$, then $G \in \mathscr{J}$. 
Proof. Since $H$ is a bounded holomorphic function, $\gamma_{1}=\lim _{r \rightarrow 1} \int_{r \mathbb{S}^{1}} \log |H| d m$ is finite. The condition $\lambda_{G} \geq|H| \lambda_{\mathbb{D}}$ implies that the zeros of $G^{\prime}$ form a Blaschke sequence, which in turn implies that the integral $\gamma_{2}=\lim _{r \rightarrow 1} \int_{r \mathbb{S}^{1}} \log \left|G^{\prime}\right| d m$ is also finite. An inspection of the inequality

$$
0 \leq \liminf _{r \rightarrow 1} \int_{r \mathbb{S}^{1}} \log \frac{\lambda_{G}}{|H| \lambda_{\mathbb{D}}} d m \leq-\gamma_{1}+\gamma_{2}-\limsup _{r \rightarrow 1} \int_{r \mathbb{S}^{1}} \log ^{+}\left|G^{\prime}\right| d m
$$

then shows that $G^{\prime}$ satisfies the Nevanlinna condition (3.1). It remains to prove that the outer part of $G$ is trivial, so that $G$ is an inner function. If this were not the case, then for a positive measure set of directions $\theta \in[0,2 \pi), \limsup _{r \rightarrow 1} \lambda_{G}\left(r e^{i \theta}\right)$ would be finite. However, this contradicts the assumption $\lambda_{G} \geq|H| \lambda_{\mathbb{D}}$, since by the LusinPrivalov theorem, the radial limit of $H\left(r e^{i \theta}\right)$ is non-zero almost everywhere.

\subsection{Injectivity and minimality}

We now show the injectivity part of Theorem 1.1. If there were two functions $F, G \in$ $\mathscr{J}$ with $\operatorname{Inn} F^{\prime}=\operatorname{Inn} G^{\prime}=B_{C} S_{\mu}$, then

$$
\lambda_{F} \geq \lambda_{F \wedge G} \geq\left|B_{C} S_{\mu}\right| \cdot \lambda_{\mathbb{D}} .
$$

Lemmas 3.4 and 3.5 imply that $(F \wedge G)^{\prime}$ has the same inner part as $F^{\prime}$. From the definition of curvature, $\Delta \log \left(\lambda_{F} / \lambda_{F \wedge G}\right)=4\left(\lambda_{F}^{2}-\lambda_{F \wedge G}^{2}\right)$. Hence $\log \left(\lambda_{F} / \lambda_{F \wedge G}\right)$ is subharmonic and non-negative, yet

$$
\lim _{r \rightarrow 1} \int_{|z|=r} \log \left(\lambda_{F} / \lambda_{F \wedge G}\right) d m \rightarrow 0
$$

which forces $\log \left(\lambda_{F} / \lambda_{F \wedge G}\right)=0$. We deduce that $\lambda_{F}=\lambda_{F \wedge G}=\lambda_{G}$ and therefore $F=G$ up to post-composition with a Möbius transformation by [15, Theorem 5.1].

Minimality is similar. Given an inner function $F \in \mathscr{J}$, we now show that $\lambda_{F}$ is the smallest metric of constant curvature -4 that exceeds $\left|\operatorname{Inn} F^{\prime}\right| \lambda_{\mathbb{D}}$. Following Section 2.2, we consider the hull $\lambda$ of the metric $\left|\operatorname{Inn} F^{\prime}\right| \lambda_{\mathbb{D}}$. The inequalities

$$
\lambda_{F} \geq \lambda \geq\left|\operatorname{Inn} F^{\prime}\right| \lambda_{\mathbb{D}}
$$

reveal that $\lambda$ has exactly the same zero set as $\lambda_{F}$ (counted with multiplicity). In particular, all zeros of $\lambda$ have integral multiplicities. Proceeding like in the proof of injectivity, we obtain $\lim _{r \rightarrow 1} \int_{|z|=r} \log \left(\lambda_{F} / \lambda\right) d m \rightarrow 0$ and $\lambda=\lambda_{F}$ as desired. 


\section{Stable approximations}

In this section, we study approximations of inner functions by finite and maximal Blaschke products. We are particularly interested in stable approximations where the inner-outer decomposition is preserved in the limit:

Definition. Suppose $\left\{F_{n}\right\} \subset \mathscr{J}$ is a sequence of inner finctions which converge uniformly on compact subsets of the disk to an inner function $F$. We say that $F_{n}$ is a (Nevanlinna) stable approximation of $F$ if

$$
\operatorname{Inn} F^{\prime}=\lim _{n \rightarrow \infty} \operatorname{Inn} F_{n}^{\prime}, \quad \text { Out } F^{\prime}=\lim _{n \rightarrow \infty} \text { Out } F_{n}^{\prime}
$$

In general, we have inequalities in one direction:

Theorem 4.1. Suppose $\left\{F_{n}\right\} \subset \mathscr{J}$ is a sequence of finite Blaschke products which converge uniformly on compact subsets of the disk to a holomorphic function $F$ : $\mathbb{D} \rightarrow \mathbb{D}$. Also assume that the $B_{n}=\operatorname{Inn} F_{n}^{\prime}$ converge to an inner function $I$. Then $F \in \mathscr{J}$ and the following inequalities hold:

$$
\begin{aligned}
\sigma\left(F^{\prime}\right) & \leq \sigma(I), \\
\left|\operatorname{Inn} F^{\prime}\right| & \geq|I|, \\
\int_{\mathbb{S}^{1}} \log \left|F^{\prime}\right| d m & \leq \lim _{n \rightarrow \infty} \int_{\mathbb{S}^{1}} \log \left|F_{n}^{\prime}\right| d m .
\end{aligned}
$$

Furthermore, either all of the above inequalities are equalities or none of them are.

Proof. Step 1. Taking $n \rightarrow \infty$ in $\lambda_{F_{n}} \geq\left|B_{n}\right| \lambda_{\mathbb{D}}$ gives $\lambda_{F} \geq|I| \lambda_{\mathbb{D}}$. Lemma 3.6 shows that $F$ is inner with $F^{\prime} \in \mathcal{N}$. For any $0<r<1$ and interval $E \subset \mathbb{S}^{1}$, we have

$$
\int_{r E} \log \frac{\lambda_{F}}{\lambda_{\mathbb{D}}} d m \geq \int_{r E} \log |I| d m
$$

Taking $r \rightarrow 1$ and using Lemma 3.1 as well as the easy part of Lemma 3.3 shows

$$
-\sigma\left(F^{\prime}\right)(E)=-\int_{E} \log \left|F^{\prime}\right| d m+\lim _{r \rightarrow 1} \int_{r E} \log \left|F^{\prime}\right| d m \geq-\sigma(I)(E),
$$

provided the endpoints of $E$ do not charge $\sigma(I)$ and $\sigma\left(F^{\prime}\right)$. This proves the first inequality 4.2 . 
Step 2. Clearly, $\operatorname{Inn} F^{\prime}$ and $I=\lim \left(\operatorname{Inn} F_{n}^{\prime}\right)$ have the same zeros in the unit disk but may have different singular factors. However, it is easy to see that for singular inner functions, one has the inequality $S_{1} \leq S_{2}$ if and only if $\sigma\left(S_{1}\right) \geq \sigma\left(S_{2}\right)$. The "if" direction is obvious, while the "only if" direction follows from the identity

$$
0 \leq \lim _{r \rightarrow 1} \int_{r E} \log \left|S_{2} / S_{1}\right| d m=-\sigma\left(S_{2}\right)(E)+\sigma\left(S_{1}\right)(E),
$$

valid for any generic interval $E \subset \mathbb{S}^{1}$ whose endpoints do not charge the measures $\sigma\left(S_{1}\right)$ and $\sigma\left(S_{2}\right)$. This proves (4.3) and shows that the equality cases in (4.2) and (4.3) coincide.

Since $F_{n}^{\prime} \rightarrow F^{\prime}$ uniformly on compact subsets of the disk, (4.3) is equivalent to the inequality $\left|\operatorname{Out} F^{\prime}(z)\right| \leq \mid \lim _{n \rightarrow \infty}$ Out $F_{n}^{\prime}(z) \mid$. Setting $z=0$ and taking logarithms gives (4.4). However, if (4.4) is an equality, then by the maximum modulus principle, we must have $\mid$ Out $F^{\prime}(z)|=| \lim _{n \rightarrow \infty}$ Out $F_{n}^{\prime}(z) \mid$ for all $z \in \mathbb{D}$, since outer factors do not vanish. This completes the proof.

Remark. After we prove the fundamental lemma (Lemma 2.3), the assumption that the $F_{n}$ be finite Blaschke products in Theorem 4.1 will no longer be necessary.

For some applications, we need to slightly vary the assumptions in the above theorem:

Theorem 4.2. In the context of Theorem 4.1, suppose instead that the $B_{n}$ converge to a non-zero holomorphic function $H: \mathbb{D} \rightarrow \mathbb{D}$ with the inner-outer decomposition $H=I \cdot O$. Then, the inequalities (4.2) (4.4) still hold. The equality case in (4.4) implies that $\left\{F_{n}\right\}$ is a stable sequence, in particular, the outer factor $O=1$ is trivial and the $B_{n}$ converge to an inner function.

The proof of 4.2 is nearly identical to that of Theorem 4.1, so we only sketch the details. Observe that since $\|H\|_{\infty} \leq 1$, we have $|O(z)| \leq 1$ and $|I(z)| \geq|H(z)|$ for $z \in \mathbb{D}$. Following Step 1 of the proof of Theorem 4.1, we obtain the inequality $\lambda_{F} \geq|I \cdot O| \lambda_{\mathbb{D}}$. We may still use Lemma 3.6 to conclude that $F \in \mathscr{J}$. The remark after Lemma 3.4 allows us to conclude 4.2 and 4.3 in this more general case as well. We may weaken (4.3) to $\left|\operatorname{Inn} F^{\prime}\right| \geq|H|$, which is equivalent to (4.4). This time, the equality case in (4.4) forces $I=H$ and $O=1$. 
Remark. In view of Lemma 3.6, if a sequence of finite Blaschke products $F_{n}$ converges to a function $F \notin \mathcal{N}$, then $H=\lim \left(\operatorname{Inn} F_{n}^{\prime}\right)$ must be 0 .

Craizer's argument from [4, Lemma 5.4] shows:

Lemma 4.3. Any inner function $F \in \mathscr{J}$ admits a stable approximation.

Proof. Suppose $\xi \in \mathbb{D}$ is such that $T_{\xi} \circ F$ is a Blaschke product, where $T_{\xi}=\frac{z-\xi}{1-\bar{\xi} z}$. We may choose a sequence $F_{n, \xi}$ of finite Blaschke products converging to $F$ so that $T_{\xi} \circ F_{n, \xi}$ is a sequence of partial products of $T_{\xi} \circ F$. Then, for any $x \in \mathbb{S}^{1}$, we have

$$
\left|\left(T_{\xi} \circ F_{n, \xi}\right)^{\prime}(x)\right| \leq\left|\left(T_{\xi} \circ F\right)^{\prime}(x)\right|
$$

see [18, Corollary 4.13]. It follows that

$$
\left|\left(F_{n, \xi}\right)^{\prime}(x)\right| \leq\left[\frac{1+|\xi|}{1-|\xi|}\right]^{2}\left|F^{\prime}(x)\right|
$$

which leads to the estimate

$$
\int_{\mathbb{S}^{1}} \log \left|F_{n, \xi}^{\prime}(x)\right| d m \leq 2 \log \frac{1+|\xi|}{1-|\xi|}+\int_{\mathbb{S}^{1}} \log \left|F^{\prime}(x)\right| d m .
$$

Since we can choose $\xi$ arbitrarily close to 0 (e.g. see [18, Theorem 2.5]), we can diagonalize to find a sequence $F_{n}$ converging to $F$ for which

$$
\limsup _{n \rightarrow \infty} \int_{\mathbb{S}^{1}} \log \left|F_{n}^{\prime}(x)\right| d m \leq \int_{\mathbb{S}^{1}} \log \left|F^{\prime}(x)\right| d m .
$$

However, by Theorem 4.2 , the lower bound is automatic and the sequence $\left\{F_{n}\right\}$ is stable.

Remark. Since translation $f \rightarrow T_{\xi} \circ f$ is continuous in $L^{1}\left(\mathbb{S}^{1}\right)$, the above proof shows that $\log \left|F_{n}^{\prime}\right| \rightarrow \log \left|F^{\prime}\right|$ converges in the $L^{1}$-norm.

With the help of a Nevanlinna stable approximation $F_{n} \rightarrow F$, we can deduce 2.2 by taking $n \rightarrow \infty$ in $\lambda_{F_{n}} \geq\left|\operatorname{Inn} F_{n}^{\prime}\right| \lambda_{\mathbb{D}}$. Since minimality was proved in Section 3.3 , the proof of the fundamental lemma (Lemma 2.3) is complete.

We can endow the space of analytic functions $\mathscr{E}=\left\{f: f^{\prime} \in \mathcal{N}\right\}$ with the strong stable topology by specifying that $f_{n} \rightarrow f$ if the $f_{n}$ converge uniformly on compact sets 
to $f$ and $\log \left|f_{n}^{\prime}\right| \rightarrow \log \left|f^{\prime}\right|$ in the $L^{1}\left(\mathbb{S}^{1}\right)$-norm. By the above remark, finite Blaschke products are dense in $\mathscr{J}$, while Theorem 4.1 implies that the subset $\mathscr{J} \subset \mathscr{E}$ is closed (see the remark after the theorem). Another possible topology on $\mathscr{E}$ is the weak stable topology where one only requires the weak-* convergence of measures $\log \left|f_{n}^{\prime}\right| d m \rightarrow \log \left|f^{\prime}\right| d m$. The above properties also hold in this topology.

\subsection{Example of an unstable approximation}

We now give an example of a sequence of finite Blaschke products which is not Nevanlinna stable. Let $F_{n}$ be the Blaschke product of degree $n+1$ with zeros at the origin and at $z_{j}=e^{j(2 \pi i / n)} \cdot\left(1-1 / n^{2}\right), j=1,2, \ldots, n$. With the normalization $F_{n}^{\prime}(0)>0$, the maps $F_{n}$ converge to the identity since $\sum_{j=1}^{n}\left(1-\left|z_{j}\right|\right) \rightarrow 0$ as $n \rightarrow \infty$. Recall that for $x \in \mathbb{S}^{1}$, one has the formula $\left|F_{n}^{\prime}(x)\right|=1+\sum_{j=1}^{n} P_{z_{j}}(x)$, where $P_{z}$ is the Poisson kernel as viewed from $z \in \mathbb{D}$, e.g. see [18, Theorem 4.15]. Computations show

$$
\int_{I_{j}} \log \left|F_{n}^{\prime}\right| d m \geq \int_{I_{j}} \log \left|1+P_{z_{j}}\right| d m \gtrsim 1 / n
$$

where $I_{j}$ consists of the points on the unit circle for which the closest zero is $z_{j}$. Hence, $\mid$ Out $F_{n}^{\prime}(0)\left|=\exp \int_{\mathbb{S}^{1}} \log \right| F_{n}^{\prime} \mid d m>c>1$ for some constant $c$ independent of $n \geq 1$. Since the outer parts Out $F_{n}^{\prime}$ do not converge to the constant function 1 , neither can the inner parts Inn $F_{n}^{\prime}$.

A modification of this construction can be used to show the existence of a sequence of finite Blaschke products $F_{n} \rightarrow z$ (and thus $F_{n}^{\prime} \rightarrow 1$ ) for which $\operatorname{Inn} F_{n}^{\prime} \rightarrow S_{\delta_{1}}$ and Out $F_{n}^{\prime} \rightarrow 1 / S_{\delta_{1}}$.

\section{Understanding the image}

In this section, we discuss the image of the map $F \rightarrow \operatorname{Inn} F^{\prime}$ and prove the decomposition, product and division rules from the introduction. We also show that the map $F \rightarrow \operatorname{Inn} F^{\prime}$ is not surjective by exhibiting a large class of invisible measures. A complete description of the image will be given in the next section. 


\subsection{Wedging $F_{\mu}$ with $F_{C}$}

Theorem 5.1. (i) Suppose $F_{\mu} \in \mathscr{J}$ is an inner function with $\operatorname{Inn} F_{\mu}^{\prime}=S_{\mu}$. Let $F_{\mu, C}=F_{\mu} \wedge F_{C}$ where $C$ is a Blaschke sequence. Then, Inn $F_{\mu, C}^{\prime}=B_{C} S_{\mu}$.

(ii) Conversely, if $F_{\mu, C} \in \mathscr{J}$ is an inner function with $\operatorname{Inn} F_{\mu, C}^{\prime}=B_{C} S_{\mu}$, then there exists an inner function $F_{\mu}$ with $\operatorname{Inn} F_{\mu}^{\prime}=S_{\mu}$.

Proof. (i) Since $\lambda_{F_{C}} \geq \lambda_{F_{\mu, C}} \geq\left|B_{C}\right| \lambda_{F_{\mu}}$, the critical set of $F_{\mu, C}$ is precisely $C$ with the correct multiplicity; while the inequalities $\lambda_{F_{\mu}} \geq \lambda_{F_{\mu, C}} \geq\left|B_{C}\right| \lambda_{F_{\mu}}$ show that $\sigma\left(F_{\mu, C}^{\prime}\right)=\mu$. Hence $\operatorname{Inn} F_{\mu, C}^{\prime}=B_{C} S_{\mu}$ as desired.

(ii) Suppose $F_{\mu, C} \in \mathscr{J}$ is an inner function with $\operatorname{Inn} F_{\mu, C}^{\prime}=B_{C} S_{\mu}$. Let $F_{n}$ be some approximation of $F_{\mu, C}$ by finite Blaschke products (stability is not required in this proof). For any $0<r<1$, we can form the sequence of finite Blaschke products $F_{n, r}$ by removing the critical points from $F_{n}$ that lie in the ball $\{z:|z|<r\}$, and considering the maximal Blaschke product with the remaining critical points (with the normalization $F_{n, r}(0)=0$ and $\left.F_{n, r}^{\prime}(0)>0\right)$. For each $r$, we pick a subsequential limit $F_{r}$ of $F_{n, r}$. We may then extract a further subsequential limit $F$ by taking $r \rightarrow 1$. By construction, we have

$$
\left|B_{C}\right| \lambda_{F} \leq \lambda_{F_{\mu, C}} \leq \lambda_{F}
$$

Since the limit $F$ cannot be constant, by Hurwitz' theorem, $F$ has no critical points. The above inequalities imply $\sigma\left(F^{\prime}\right)=S_{\mu}$ and therefore Inn $F^{\prime}=S_{\mu}$.

\subsection{Subseqences of stable sequences}

In the next lemma, we show that any subsequence of a stable sequence is also stable:

Lemma 5.2. Suppose that $F_{C_{n}} \rightarrow F_{\mu_{1}+\mu_{2}}$ is a stable sequence. Suppose that $C_{1, n} \subset$ $C_{n}$ is such that $B_{C_{1, n}}$ converges to $S_{\mu_{1}}$. Then, $F_{C_{1, n}} \rightarrow F_{\mu_{1}}$.

Proof. Write $C_{n}=C_{1, n} \cup C_{2, n}$. From the assumptions, $B_{C_{1, n}} \rightarrow S_{\mu_{1}}$ and $B_{C_{2, n}} \rightarrow S_{\mu_{2}}$. After passing to a subsequence, we can ensure convergence:

$$
F_{C_{1, n}} \rightarrow F_{\nu_{1}}, \quad \nu_{1} \leq \mu_{1}
$$




$$
F_{C_{2, n}} \rightarrow F_{\nu_{2}}, \quad \nu_{2} \leq \mu_{2}
$$

The monotonicity of limits follows from Theorem 4.1 .

For each $n$, we have $\lambda_{F_{C_{n}}} \geq\left|B_{1, n}\right| \lambda_{F_{C_{2, n}}}$ and therefore, after taking $n \rightarrow \infty$, we see that

$$
\lambda_{F_{\mu_{1}+\mu_{2}}} \geq\left|S_{\mu_{1}}\right| \lambda_{F_{\nu_{2}}} .
$$

As is now standard, we may deduce

$$
\mu_{1}+\mu_{2} \leq \mu_{1}+\nu_{2}
$$

by examining the equation

$$
0 \leq \lim _{r \rightarrow 1} \int_{r I} \log \frac{\lambda_{F_{\mu_{1}+\mu_{2}}}}{\left|S_{\mu_{1}}\right| \lambda_{F_{\nu_{2}}}} d m
$$

Hence $\nu_{2}=\mu_{2}$ (and similarly $\left.\nu_{1}=\mu_{1}\right)$ as desired.

The above lemma has a number of consequences:

Corollary 5.3. If a measure $\mu$ is constructible, i.e. if $F_{\mu}$ exists, then all $\nu \leq \mu$ are also constructible. Combining with Theorem 5.1, we see that the image of the mapping $F \rightarrow \operatorname{Inn} F^{\prime}$ is closed under taking divisors.

Indeed, given a stable approximation $F_{C_{n}}$ to $F_{\mu}$, it is not difficult to select $C_{1, n} \subset$ $C_{n}$ so that $B_{C_{1, n}} \rightarrow S_{\nu}$

Corollary 5.4. If $F_{\mu_{1}}$ and $F_{\mu_{2}}$ are constructible, then $F_{\mu_{1}+\mu_{2}}$ is also constructible.

The proof relies on the Solynin-type estimate

$$
\lambda_{F_{C_{1}}} \lambda_{F_{C_{2}}} \geq \lambda_{F_{C_{1} \cup C_{2}}} \lambda_{F_{C_{1} \cap C_{2}}}
$$

valid when $C_{1}$ and $C_{2}$ are finite subsets of the disk counted with multiplicity. The proof of (5.1) is essentially that of [16, Lemma 2.8], so we only sketch the details. Consider the function

$$
u(z)=\log ^{+}\left(\frac{\lambda_{F_{C_{1} \cup C_{2}}} \lambda_{F_{C_{1} \cap C_{2}}}}{\lambda_{F_{C_{1}}} \lambda_{F_{C_{2}}}}\right), \quad z \in \mathbb{D} .
$$


We claim that it is subharmonic and non-negative in $\mathbb{D}$ yet tends to 0 as $|z| \rightarrow 1$. This will show that it is equal to 0 identically. It is clearly non-negative by definition. To show that $u(z)$ is subharmonic, one can check that $\Delta u \geq 0$. We refer the reader to [16, Lemma 2.8] for the computation. For the last statement, note that by Lemma 2.3 . for a finite Blaschke product, the quotient $\lambda_{F} / \lambda_{\mathbb{D}} \rightarrow 1$ uniformly as $|z| \rightarrow 1$.

Proof of Corollary 5.4. Choose approximations $F_{C_{1, n}} \rightarrow F_{\mu_{1}}$ and $F_{C_{2, n}} \rightarrow F_{\mu_{2}}$ by finite Blaschke products. Making a small perturbation if necessary, we can assume that the sets $C_{1, n}$ and $C_{2, n}$ are disjoint. Let $C_{n}=C_{1, n} \cup C_{2, n}$ be their union. Passing to a subsequence, we may assume that $F_{C_{n}} \rightarrow F_{\mu}$ for some measure $\mu$ on the unit circle. By Solynin's estimate, we have

$$
\log \frac{\lambda_{\mathbb{D}}}{\lambda_{F_{C_{1, n}}}}+\log \frac{\lambda_{\mathbb{D}}}{\lambda_{F_{C_{2, n}}}} \leq \log \frac{\lambda_{\mathbb{D}}}{\lambda_{F_{C_{n}}}} .
$$

Taking $n \rightarrow \infty$ gives

$$
\log \frac{\lambda_{\mathbb{D}}}{\lambda_{F_{\mu_{1}}}}+\log \frac{\lambda_{\mathbb{D}}}{\lambda_{F_{\mu_{2}}}} \leq \log \frac{\lambda_{\mathbb{D}}}{\lambda_{F_{\mu}}} .
$$

By examining averages over $r I$ and taking $r \rightarrow 1$, we discover that $\mu \geq \mu_{1}+\mu_{2}$. Applying Corollary 5.3 shows that the measure $\mu_{1}+\mu_{2}$ is constructible.

Corollary 5.5. If $S_{\mu}^{\prime} \in \mathcal{N}$ then $\mu$ is constructible.

In [3], M. Cullen showed that this is the case when the support of $\mu$ is a BeurlingCarleson set, that is, a closed subset of the unit circle of zero Lebesgue measure whose complement is a union of $\operatorname{arcs} \bigcup_{k} I_{k}$ with $\sum\left|I_{k}\right| \log \frac{1}{\left|I_{k}\right|}<\infty$.

\subsection{Invisible measures}

Let $\mu$ be a finite positive measure on the unit circle, which is singular with respect to the Lebesgue measure. We say $\mu$ is invisible if for any measure $0<\nu \leq \mu$, there does not exist a function $F_{\nu} \in \mathscr{J}$ with $\operatorname{Inn} F_{\nu}^{\prime}=S_{\nu}$.

Lemma 5.6. Either the map $F \rightarrow \operatorname{Inn} F^{\prime}$ is surjective or there exists an invisible measure. 
Proof. Suppose $F_{\mu}$ is not constructible. Since the hull of the metric $\left|S_{\mu}\right| \cdot \lambda_{\mathbb{D}}$ defined in Section 2.2 cannot vanish anywhere, it must be of the form $\lambda_{F_{\nu}}$ for some measure $\nu$. (Lemma 3.6 explains why $F_{\nu}$ must be an inner function.) Applying Lemma 3.4 . we see that $\nu<\mu$ since equality cannot hold. From the product rule (Corollary 5.4), it follows that the measure $\mu-\nu$ is invisible. More precisely, if $\sigma \leq \mu-\nu$ was constructible, then $\lambda_{F_{v}}>\lambda_{F_{\mu-\sigma / 2}}>\left|S_{\mu}\right| \cdot \lambda_{\mathbb{D}}$ would contradict the definiton of $\nu$.

Actually, the above argument shows a little more:

Theorem 5.7. A measure $\mu$ is invisible if and only if the hull of $\left|S_{\mu}\right| \cdot \lambda_{\mathbb{D}}$ is the Poincaré metric. More generally, any measure $\mu$ can be uniquely decomposed into a constructible part and an invisible part: $\mu=\mu_{\mathrm{con}}+\mu_{\mathrm{inv}}$, in which case, the hull of $\left|S_{\mu}\right| \cdot \lambda_{\mathbb{D}}$ is $\lambda_{F_{\mu_{\mathrm{con}}}}$.

We are now in a position to prove the countable version of the product rule (Lemma 1.3). Suppose we are given countably many constructible measures $\mu_{j}$, $j=1,2, \ldots$ such that their their sum $\mu=\sum_{j=1}^{\infty} \mu_{j}$ is a finite measure. We claim that $\mu$ is constructible. According to Theorem 5.7, the hull of $\left|S_{\mu}\right| \cdot \lambda_{\mathbb{D}}$ is of the form $\lambda_{F_{\nu}}$ for some measure $\nu \leq \mu$. However, from Corollary 5.4, we know that $\tilde{\mu}_{j}=\mu_{1}+\mu_{2}+\cdots+\mu_{j}$ is constructible. This shows that $\nu \geq \tilde{\mu}_{j}$ for any $j$, which forces $\nu=\mu$.

\subsection{A criterion for invisibility}

In this section, we only consider conformal metrics with strictly positive densities, that is, genuine metrics instead of pseudometrics. Given a positive continuous function $u$ on $\mathbb{S}_{r}=\{z:|z|=r\}, 0<r<1$, let $\Lambda_{r}[u]$ denote the unique conformal metric of curvature -4 on $\mathbb{D}_{r}=\{z:|z|<r\}$ which agrees with $u$ on $\mathbb{S}_{r}$. For the existence and uniqueness of $\Lambda_{r}[u]$, we refer the reader to [10, Section 12] or [15, Appendix]. For a non-vanishing SK-metric $\lambda$, we will sometimes write $\Lambda[\lambda]=\hat{\lambda}$ for the minimal metric of curvature -4 that exceeds $\lambda$.

Lemma 5.8. The operation $u \rightarrow \Lambda_{r}[u]$ is monotone in $u$, that is, if $u \geq v$ then $\Lambda_{r}[u] \geq \Lambda_{r}[v]$. 
To see this, note that the function $h=\log ^{+}\left(\Lambda_{r}[v] / \Lambda_{r}[u]\right)$ is non-negative, subharmonic and identically zero on $\mathbb{S}_{r}$. As usual, to check that $h$ is subharmonic, we use the definition of curvature:

$$
\Delta h=\left(4 \Lambda_{r}[v]^{2}-4 \Lambda_{r}[u]^{2}\right) \cdot \chi_{v>u} \geq 0 .
$$

A similar argument shows:

Lemma 5.9. Let $\lambda$ be a non-vanishing conformal metric on the unit disk of curvature at most -4 . For $0<r<1$, the metric $\Lambda_{r}\left[\lambda\left(r e^{i \theta}\right)\right]$ is the minimal metric of curvature -4 that exceeds $\lambda$ on $\mathbb{D}_{r}$. The family of metrics $\Lambda_{r}\left[\lambda\left(r e^{i \theta}\right)\right]$ is non-decreasing in $r$, and the limit

$$
\hat{\lambda}=\Lambda[\lambda]=\lim _{r \rightarrow 1} \Lambda_{r}\left[\lambda\left(r e^{i \theta}\right)\right]
$$

is the minimal metric of curvature -4 that exceeds $\lambda$ on $\mathbb{D}$.

In general, it is difficult to evaluate $\Lambda_{r}[u]$ explicitly. In the next lemma, we do so when $u$ is a constant function.

Lemma 5.10. Given any $0<c \leq 1$, there exists a unique $0<r^{\prime} \leq r$ so that $\Lambda_{r}\left[c \cdot \lambda_{\mathbb{D}}\right]=L^{*} \lambda_{\mathbb{D}}$ where $L(z)=\frac{r^{\prime}}{r} \cdot z$ is the linear map $\mathbb{D}_{r} \rightarrow \mathbb{D}_{r^{\prime}}$.

The lemma follows by observing that the metrics $\left(L_{r^{\prime}}\right)^{*} \lambda_{\mathbb{D}}$ are increasing in $r^{\prime}$, so there is a unique value of $r^{\prime}$ to make the boundary values agree.

Corollary 5.11. We have

$$
\lim _{C \rightarrow \infty}\left[\lim _{r \rightarrow 1} \frac{\Lambda_{r}[C]}{\lambda_{\mathbb{D}}}\right] \rightarrow 1,
$$

uniformly on compact subsets of the unit disk.

With these preparations, we can now prove:

Theorem 5.12. Suppose $\mu$ is a singular measure on the unit circle which satisfies $\mu(I) \leq C|I| \log |1 / I|$ for any interval $I \subset \mathbb{S}^{1}$ and some constant $C>0$. Then, $\mu$ is invisible. 
Proof. From the product rule (Corollary 5.4), it is easy to see that a measure $\mu$ is invisible if and only if $\varepsilon \cdot \mu$ is for any $\varepsilon>0$. This allows us to assume that $\mu(I) \leq$ $\varepsilon|I| \log |1 / I|$ which implies that the Poisson extension $P_{\mu}(z) \leq \varepsilon\left(A \log \frac{1}{1-|z|}+B\right)$ for some constants $A$ and $B$. Hence, $\left|S_{\mu}\right| \lambda_{\mathbb{D}} \rightarrow \infty$ as $|z| \rightarrow 1$. The theorem now follows from the monotonicity principle (Lemma 5.8) and Corollary 5.11.

\section{Roberts decompositions}

In this section, we show that if $\mu$ does not charge Beurling-Carleson sets, then it is invisible, that is, any measure $0<\nu \leq \mu$ cannot be in the image of the map $F \rightarrow \operatorname{Inn} F^{\prime}$. To upgrade the argument of Section 5.4, we will use the following theorem which is implicit in the work of Roberts [19]:

Theorem 6.1. Suppose $\mu$ is a measure on the unit circle which does not charge Beurling-Carleson sets. Given a real number $c>0$ and integer $j_{0} \geq 1, \mu$ can be expressed as a countable sum

$$
\mu=\sum_{j=1}^{\infty} \mu_{j},
$$

where each $\mu_{j}$ enjoys an estimate on the modulus of continuity:

$$
\omega_{\mu_{j}}\left(1 / n_{j}\right) \leq \frac{c}{n_{j}} \cdot \log n_{j}, \quad n_{j}:=2^{2^{j+j_{0}}}
$$

Here, $\omega_{\mu}(t)=\sup _{I \subset \mathbb{S}^{1}} \mu(I)$, with the supremum taken over all intervals of length $t$.

It will be important for us that the measure $\mu$ admits infinitely many decompositions with different parameters $c$ and $j_{0}$, where $c$ can be made arbitrarily small and $j_{0}$ arbitrarily large.

Sketch of proof. For each $j=1,2, \ldots$, we can define a partition $P_{j}$ of the unit circle into $n_{j}$ equal arcs. Since $n_{j}$ divides $n_{j+1}$, each next partition can be chosen to be a refinement of the previous one. Given any measure $\mu$ on the unit circle, Roberts defines the notion of the grating of $\mu$ with respect to the sequence of partitions $\left(P_{j}\right)$.

This procedure decomposes $\mu=\sum_{j=1}^{\infty} \mu_{j}+\nu$ so that 6.2 holds for each $j$, with 
the residual measure $\nu$ supported on the union of a Beurling-Carleson set and a countable set.

To define $\mu_{1}$, consider all intervals in the partition $P_{1}$. Define an interval to be light if $\mu(I) \leq\left(c / n_{1}\right) \cdot \log n_{1}$ and heavy otherwise. On a light interval, take $\mu_{1}=\mu$, while on a heavy interval, let $\mu_{1}$ be a multiple of $\mu$ so that the mass $\mu_{1}(I)=\left(c / n_{1}\right) \cdot \log n_{1}$. Clearly, $\mu_{1}<\mu$. Consider the difference $\mu-\mu_{1}$ and grate it with respect to partition $P_{2}$ to form the measure $\mu_{2}$, then consider $\mu-\mu_{1}-\mu_{2}$ and grate it with respect to $P_{3}$ to form $\mu_{3}$, and so on. Continuing in this way, we obtain a sequence of measures $\mu_{1}, \mu_{2}, \ldots$ where each next measure is supported on the heavy intervals of the previous generation.

By construction, the bound (6.2) holds for all $j$. Inspection reveals that the residual measure $\nu$ is supported on the set of points which lie in heavy intervals at every stage. Up to a countable set, this coincides with $\mathbb{S}^{1} \backslash \mathscr{L}$, where $\mathscr{L}$ is the union of the light intervals of any generation. (This countable set consists of points on the unit circle which are endpoints of two different light intervals.) In [19, Proof of Theorem 2], Roberts gave a simple computation using the relation $\log n_{j+1}=2 \log n_{j}$ to show that $\mathbb{S}^{1} \backslash \mathscr{L}$ is a Beurling-Carleson set.

Now, if $\mu$ does not charge Beurling-Carleson sets, it does not charge points so it cannot charge countable sets, which forces the residual measure to be 0 .

The estimate 6.2 on the modulus of continuity is easily seen to be equivalent to an estimate on the Poisson extension:

$$
\left|P_{\mu_{j}}\right| \leq c^{\prime} \cdot \log \frac{1}{1-|z|^{2}}, \quad z \in B\left(0,1-1 / n_{j}\right) .
$$

Here, the constant $c^{\prime}$ can be taken to be $c c_{1}$ for some $c_{1}>0$. This is stated in [19, Lemma 2.2].

We will also need a simple lemma on conformal metrics:

Lemma 6.2. (i) For any two singular measures $\mu_{1}$ and $\mu_{2}$ on the unit circle,

$$
\Lambda\left[\left|S_{\mu_{1}}\right| \cdot \Lambda\left[\left|S_{\mu_{2}}\right| \lambda_{\mathbb{D}}\right]\right]=\Lambda\left[\left|S_{\mu_{1}}\right|\left|S_{\mu_{2}}\right| \cdot \lambda_{\mathbb{D}}\right]
$$

(ii) More generally,

$$
\Lambda\left[\left|S_{\mu_{1}}\right| \cdot \ldots \Lambda\left[\left|S_{\mu_{j-1}}\right| \cdot \Lambda\left[\left|S_{\mu_{j}}\right| \lambda_{\mathbb{D}}\right]\right] \ldots\right]=\Lambda\left[\left|S_{\mu_{1}}\right|\left|S_{\mu_{2}}\right| \cdots\left|S_{\mu_{j}}\right| \cdot \lambda_{\mathbb{D}}\right]
$$


(iii) For $\mu=\sum_{j=1}^{\infty} \mu_{j}$, we have

$$
\lim _{n \rightarrow \infty} \Lambda\left[\left|S_{\mu_{1}}\right| \cdot \ldots \Lambda\left[\left|S_{\mu_{j-1}}\right| \cdot \Lambda\left[\left|S_{\mu_{j}}\right| \lambda_{\mathbb{D}}\right]\right] \ldots\right]=\Lambda\left[\left|S_{\mu}\right| \lambda_{\mathbb{D}}\right] .
$$

Proof. (i) The $\geq$ direction follows from the monotonicity of $\Lambda$. For the $\leq$ direction, it suffices to show that

$$
\left|S_{\mu_{1}}\right| \cdot \Lambda\left[\left|S_{\mu_{2}}\right| \lambda_{\mathbb{D}}\right] \leq \Lambda\left[\left|S_{\mu_{1}}\right|\left|S_{\mu_{2}}\right| \cdot \lambda_{\mathbb{D}}\right]
$$

or

$$
\left|S_{\mu_{1}}\right| \cdot \Lambda_{r}\left[\left|S_{\mu_{2}}\right| \lambda_{\mathbb{D}}\right] \leq \Lambda_{r}\left[\left|S_{\mu_{1}}\right|\left|S_{\mu_{2}}\right| \cdot \lambda_{\mathbb{D}}\right]
$$

for any $0<r<1$, cf. Lemma 5.9. To this end, we form the function

$$
u_{r}=\log ^{+}\left(\frac{\left|S_{\mu_{1}}\right| \cdot \Lambda_{r}\left[\left|S_{\mu_{2}}\right| \lambda_{\mathbb{D}}\right]}{\Lambda_{r}\left[\left|S_{\mu_{1}}\right|\left|S_{\mu_{2}}\right| \cdot \lambda_{\mathbb{D}}\right]}\right)
$$

defined on $\mathbb{D}_{r}=\{z:|z|<r\}$. Since it is subharmonic and vanishes on $\mathbb{S}_{r}=\partial \mathbb{D}_{r}$, it must be identically 0 . This proves the $\leq$ direction.

(ii) follows after applying (i) $j-1$ times.

(iii) Let $\tilde{\mu}_{j}=\mu_{1}+\mu_{2}+\cdots+\mu_{j}$. By part (i), we have

$$
\left|S_{\mu-\tilde{\mu}_{j}}\right| \cdot \Lambda\left[\left|S_{\tilde{\mu}_{j}}\right| \lambda_{\mathbb{D}}\right] \leq \Lambda\left[\left|S_{\mu}\right| \lambda_{\mathbb{D}}\right] \leq \Lambda\left[\left|S_{\tilde{\mu}_{j}}\right| \lambda_{\mathbb{D}}\right]
$$

Since $\left|S_{\mu-\tilde{\mu}_{j}}\right| \rightarrow 1$, it follows that $\Lambda\left[\left|S_{\tilde{\mu}_{j}}\right| \lambda_{\mathbb{D}}\right]$ are decreasing and converge to $\Lambda\left[\left|S_{\mu}\right| \lambda_{\mathbb{D}}\right]$. The quantities on the left side also decrease to their limit. Therefore, the limits must coincide.

With these preparations, we can now show Theorem 1.7 .

Proof of Theorem 1.7. Step 1. Let $\mu=\mu_{j}$ be the Roberts decomposition 6.1) with parameters $c$ and $j_{0}$ to be chosen later. In view of the invisibility criterion (Theorem 5.7), it suffices to show that

$$
\lambda_{j}:=\Lambda_{1-1 / n_{1}}\left[\left|S_{\mu_{1}}\right| \cdot \ldots \Lambda_{1-1 / n_{j-1}}\left[\left|S_{\mu_{j-1}}\right| \cdot \Lambda_{1-1 / n_{j}}\left[\left|S_{\mu_{j}}\right| \cdot \lambda_{\mathbb{D}}\right]\right] \ldots\right]
$$


is close to the hyperbolic metric at the origin, uniform in $j \geq 1$. Indeed, by the monotonicity properties of $\Lambda$, we have

$$
\lambda_{j} \leq \Lambda\left[\left|S_{\mu_{1}}\right| \cdot \ldots \Lambda\left[\left|S_{\mu_{j-1}}\right| \cdot \Lambda\left[\left|S_{\mu_{j}}\right| \cdot \lambda_{\mathbb{D}}\right]\right] \ldots\right],
$$

so that if $\lambda_{j}$ is close to $\lambda_{\mathbb{D}}$, then so must

$$
\Lambda\left[\left|S_{\mu_{1}}\right|\left|S_{\mu_{2}}\right| \cdots\left|S_{\mu_{n}}\right| \lambda_{\mathbb{D}}\right]
$$

Step 2. The estimate on the modulus of continuity of $\mu_{j}$ implies that $\left|S_{\mu_{j}}\right| \lambda_{\mathbb{D}} \geq$ $\lambda_{\mathbb{D}}^{4 / 5}$ on the circle $\mathbb{S}_{1-1 / n_{j}}$. Here, we use the fact that we can choose $c^{\prime}<1 / 10$ in 6.3. We claim that this implies that

$$
\Lambda_{1-1 / n_{j}}\left[\left|S_{\mu_{j}}\right| \lambda_{\mathbb{D}}\right] \geq(1 / 2) \lambda_{\mathbb{D}}, \quad \text { on } \mathbb{S}_{1-1 / n_{j-1}}
$$

Assuming (6.6), we have

$$
\left|S_{\mu_{j-1}}\right| \cdot \Lambda_{1-1 / n_{j}}\left[\left|S_{\mu_{j}}\right| \lambda_{\mathbb{D}}\right] \geq \lambda_{\mathbb{D}}^{4 / 5}, \quad \text { on } \mathbb{S}_{1-1 / n_{j-1}}
$$

We could then inductively show that $\lambda_{j} \geq(1 / 2) \lambda_{\mathbb{D}}$ on $\mathbb{S}_{1-1 / n_{1}}$. By Corollary 5.11 . this would mean that $\lambda_{j}$ is very close to $\lambda_{\mathbb{D}}$ at the origin, provided $n_{1}$ is large (this is where we use that $j_{0}$ can be made arbitrarily large.)

Step 3. Thus, we need to show that $\Lambda_{1-1 / n_{j}}\left[\left|S_{\mu_{j}}\right| \lambda_{\mathbb{D}}\right] \geq(1 / 2) \cdot \lambda_{\mathbb{D}}$ on $\mathbb{S}_{1-1 / n_{j-1}}$. Define $\varepsilon>0$ by $1-1 / n_{j}=1-\varepsilon$ so that $1-1 / n_{j-1}=1-\varepsilon^{1 / 2}$. There exists a unique $0<\ell<1$ so that $\Lambda_{1-1 / n_{j}}\left[\lambda_{\mathbb{D}}^{4 / 5}\right]=L^{*} \lambda_{\mathbb{D}}$ where $L(z)=\ell z$. Inspection shows that $1-\ell \asymp \varepsilon^{4 / 5}$. Therefore,

$$
\Lambda_{1-1 / n_{j}}\left[\left|S_{\mu_{j}}\right| \lambda_{\mathbb{D}}\right] \geq \Lambda_{1-1 / n_{j}}\left[\lambda_{\mathbb{D}}^{4 / 5}\right]=\frac{\ell}{1-|\ell z|^{2}} \geq(1 / 2) \cdot \lambda_{\mathbb{D}}, \quad \text { on } \mathbb{S}_{1-1 / n_{j-1}}
$$

as desired. 


\section{References}

[1] P. R. Ahern, D. N. Clark, On inner functions with $H_{p}$-derivative, Michigan Math. J. 21 (1974), no. 2, 115-127.

[2] A. B. Aleksandrov, J. M. Anderson, A. Nicolau, Inner functions, Bloch spaces and symmetric measures, Proc. London Math. Soc. 79 (1999), no. 2, 318-352.

[3] M. Cullen, Derivatives of singular inner functions, Michigan Math. J. 18 (1971), no. $3,283-287$.

[4] M. Craizer, Entropy of inner functions, Israel J. Math. 74 (1991), no. 2, 129-168.

[5] P. Duren, Theory of $H_{p}$ spaces, Dover Publications, 2000.

[6] K. M. Dyakonov, Smooth functions and co-invariant subspaces of the shift operator, Algebra i Analiz 4 (1992), no. 5, 117-147, in Russian. English translation: St. Petersburg Math. J. 4 (1993), no. 5, 933-959.

[7] K. M. Dyakonov, A Reverse Schwarz-Pick Inequality, Computational Methods and Function Theory 13 (2013), no. 7-8, 449-457.

[8] K. M. Dyakonov, A characterization of Möbius transformations, C. R. Math. Acad. Sci. Paris 352 (2014), no. 2, 593-595.

[9] K. M. Dyakonov, Inner functions and inner factors of their derivatives, Integr. Equ. Oper. Theory 82 (2015), no. 2, 151-155.

[10] M. Heins, On a class of conformal metrics, Nagoya Math. J. 21 (1962), 1-60.

[11] B. Korenblum, Cyclic elements in some spaces of analytic functions, Bull. Amer. Math. Soc. 5 (1981), 317-318.

[12] D. Kraus, Critical sets of bounded analytic functions, zero sets of Bergman spaces and nonpositive curvature, Proc. London Math. Soc. 106 (2013), no. 4, 931-956. 
[13] D. Kraus, O. Roth, Critical points of inner functions, nonlinear partial differential equations, and an extension of Liouville's theorem, J. London Math. Soc. 77 (2008), no. 1, 183-202.

[14] D. Kraus, O. Roth, Critical Points, the Gauss Curvature Equation and Blaschke Products, In: Blaschke Products and Their Applications, Fields Institute Communications 65 (2012), 133-157.

[15] D. Kraus, O. Roth, Conformal metrics, Lecture Notes Ramanujan Math. Society, Lecture Notes Series 19 (2013), 41-83.

[16] D. Kraus, O. Roth, Strong submultiplicativity of the Poincaré metric, J. Analysis 24 (2016), no. 1, 39-50.

[17] D. Kraus, O. Roth, Maximal Blaschke Products, Adv. Math. 241 (2013), 58-78.

[18] J. Mashreghi, Derivatives of Inner Functions, Fields Institute Monographs, 2012.

[19] J. W. Roberts, Cyclic inner functions in the Bergman spaces and weak outer functions in $H^{p}, 0<p<1$, Illinois J. Math. 29 (1985), 25-38. 